$$
\text { Conf-9505100-1 }
$$

Note: This is a draft of a paper submitted for publication. Contents of this paper should not be quoted or referred to without permission of the author(s).

To be presented at the 4th European Microbeam Analysis Society Workshop on Modern Developments and Applications in Microbeam Analysis,

St. Malo, France, May 14-19, 1995

Published in Proceedings

\title{
MICROANALYSIS AT ATOMIC RESOLUTION
}

S. J. Pennycook, D. E. Jesson, N. D. Browning, ${ }^{*}$ and M. F. Chisholm

Solid State Division, Oak Ridge National Laboratory

P.O. Box 2008, Oak Ridge, Tennessee 37831-6030

*Department of Physics (M/C 273), University of Illinois at Chicago

845 West Taylor Street, Chicago, Illinois 60607-7059

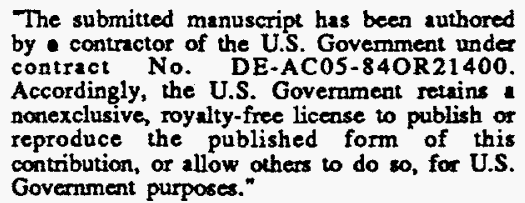

Government purposes."

Prepared by

SOLID STATE DIVISION

OAK RIDGE NATIONAL LABORATORY

Managed by

MARTIN MARIETTA ENERGY SYSTEMS, INC.

under

Contract No. DE-AC05-84OR21400

with the

U.S. DEPARTMENT OF ENERGY

Oak Ridge, Tennessee

April 1995 


\title{
Microanalysis at Atomic Resolution
}

\author{
S. J. Pennycook, D. E. Jesson, N. D. Browning ${ }^{*}$ and M. F. Chisholm
}
Solid State Division, Oak Ridge National Laboratory, P.O. Box 2008, Oak
Ridge, TN 37831-6030

\begin{abstract}
In this paper we discuss how the dedicated scanning transmission electron microscope can provide a microanalysis of crystalline materials at atomic resolution. The method requires the establishment of incoherent conditions for a reference imaging signal as well as the spectroscopic signal. The image can then be used to focus and locate the probe to atomic precision for microanalysis. The $Z$-contrast image provides the most convenient incoherent reference image, and $\mathrm{X}$-ray and electron energy loss data may be acquired simultaneously. In zone axis crystals, strong columnar channelling delays the onset of beam broadening for several hundred Angstroms, so that atomic resolution microanalysis may be achieved in materials specimens of significant thickness. This combination of signals provides a powerful means for studying interface structure and bonding, and avoids relying on preconceived model structures.

\section{DISCLAIMER}

\begin{abstract}
This report was prepared as an account of work sponsored by an agency of the United States Government. Neither the United States Government nor any agency thereof, nor any of their employees, makes any warranty, express or implied, or assumes any legal liability or responsibility for the accuracy, completeness, or usefulness of any information, apparatus, product, or process disclosed, or represents that its use would not infringe privately owned rights. Reference herein to any specific commercial product, process, or service by trade name, trademark, manufacturer, or otherwise does not necessarily constitute or imply its endorsement, recommendation, or favoring by the United States Government or any agency thereof. The views and opinions of authors expressed herein do not necessarily state or reflect those of the United States Government or any agency thereof.
\end{abstract}

* Present address: Department of Physics (M/C 273), University of Illinois at Chicago, 845 West Taylor Street, Chicago, IL 60607-7059. USA 


\section{DISCLAIMER}

Portions of this document may be illegible in electronic image products. Images are produced from the best available original document. 


\section{Introduction}

Now that the dedicated scanning transmission electron microscope (STEM) allows atomic sized probes to be routinely achieved, the potential to analyse chosen atomic columns for their composition and bonding has become a reality. Studies of materials can finally be carried out at the fundamental atomic scale. For example, impurity segregation at dislocation cores in grain boundaries can be investigated atomic column by atomic column. Such capabilities are expected to have a far-reaching impact throughout materials science [1].

In this paper we discuss the conditions under which such microanalysis is possible, including considerations of probe formation, beam broadening, and localization of spectroscopic information. The first critical requirement is the ability to form an incoherent image of the material at atomic resolution, so that the probe size and position may be accurately controlled. The Z-contrast image formed from elastically scattered electrons with a high angle annular detector is convenient for this purpose, and does not interfere with the simultaneous acquisition of $\mathrm{X}$-ray or energy loss data. Secondly, incoherent conditions for the analytical signal must be established, so that it too will show an incoherent image in direct correspondence to the Z-contrast image, although typically of much weaker intensity. In this way, the Z-contrast image can provide a high intensity reference image corresponding to the much weaker inelastic image, and can therefore be used to focus the probe to an optimum size and to locate it over particular columns of interest. A point microanalysis can therefore be achieved from specific atomic columns, in principle from a single column, provided beam damage is not too rapid. 
It is important to appreciate from the outset that atomic resolution microanalysis is only possible at present on the dedicated STEM, although there appears no reason in principle why this should not be achievable in a conventional TEM equipped with a field emission gun. The instrumental requirements are discussed further in the following section, while in section 3. we discuss the propagation of a coherent probe through a zone axis crystal. It is the remarkable behaviour of such a probe that makes atomic resolution microanalysis possible in specimens up to several hundred Angstroms in thickness. In section 4. we discuss the limits on spatial resolution imposed by the delocalization of the inelastic scattering probability. Generally, losses in excess of several hundred $\mathrm{eV}$ are required for atomic resolution to be possible, although some improvement is possible by using an annular collector aperture to exclude low-angle scattering from the spectrometer.

Finally, in section 5 we present an experimental demonstration of atomic resolution microanalysis by mapping the Co L loss intensity across an atomically abrupt $\mathrm{CoSi}_{2} / \mathrm{Si}(111)$ epitaxial interface. This intensity drops dramatically on moving from the last plane of the silicide to the first plane of the silicon, a distance of only $2.7 \AA$.

\section{Instrumental Requirements for Incoherent Imaging}

As realized first by Crewe [3,4], the high brightness of the field emission gun is essential in achieving an atomic sized probe with sufficient current for image formation. To achieve an electron-optically limited probe, a high source demagnification is used, so that the geometrical size of the source in the focal 
plane of the objective lens is much less than the actual probe size obtained by balancing the effects of spherical aberration and defocus. Under these conditions the probe is coherent, with an optimum size given by

$$
\mathrm{d}_{\mathrm{opt}}=0.43 \mathrm{C}_{\mathrm{s}}^{1 / 4} \lambda^{3 / 4}
$$

where $C_{s}$ is the objective lens spherical aberration coefficient, and $\lambda$ the electron wavelength. This optimum probe is obtained with an optimum objective aperture

$$
\alpha_{\mathrm{opt}}=\left(\frac{4 \lambda}{\mathrm{C}_{\mathrm{s}}}\right)^{1 / 4}
$$

and an optimum defocus of

$$
\Delta \mathrm{f}_{\mathrm{opt}}=-\left(\mathrm{C}_{\mathrm{s}} \lambda\right)^{1 / 2}
$$

These conditions were first identified by Scherzer [5], and later analysed in more detail by Crewe and coworkers [6,7]. Note that they differ from the more familiar Scherzer conditions for bright field phase contrast imaging. In particular, the optimum objective aperture tends to be smaller, since there is no need to include particular low order diffracted beams, and $d_{o p t}$, which is often taken as a convenient measure of microscope resolution, is significantly improved over the corresponding expression for coherent imaging,

$$
d_{\mathrm{coh}}=0.66 C_{\mathrm{s}}^{1 / 4} \lambda^{3 / 4}
$$

The higher resolution is a direct result of squaring the probe amplitude profile $\mathrm{P}(\mathbf{R})$, on which the resolution of a coherent image depends, to form 
the intensity profile $\mathrm{P}^{2}(\mathbf{R})$, which controls the resolution of an incoherent image or analysis. Probe profiles are straightforward to calculate [8], and Fig. 1. shows profiles as a function of defocus for the two STEM instruments at Oak Ridge National Laboratory, a 100 kV VG Microscopes HB501UX, with an objective lens $C_{s}$ of $1.3 \mathrm{~mm}$ giving an optimum probe size of $2.2 \AA$, and a 300 $\mathrm{kV}$ VG Microscopes HB603, with a $1 \mathrm{~mm} \mathrm{C}_{\text {s }}$ giving a $1.3 \AA$ probe. These profiles show behaviour typical of coherent probes, broad at low defocus values, becoming narrower at higher defocus but also acquiring significant subsidiary maxima. The Scherzer optimum probe provides the narrowest central peak without substantial intensity appearing in subsidiary maxima. This represents the most local probe for imaging and microanalysis.

The conditions required for incoherent imaging with elastically (including thermally) scattered electrons, and inelastically scattered electrons, have been reviewed recently [9] and will only be briefly mentioned here. Incoherent conditions apply when either all the scattering is detected corresponding to some elastic or inelastic interaction, or when a representative fraction of the total scattering is detected.

In the case of elastically scattered electrons incoherent imaging is achieved by collecting a small representative fraction of the total scattering through the use of a high angle annular detector. By ensuring that the inner angle $\theta_{i}$ of the detector is large compared to the probe forming aperture, incoherent imaging of crystal columns is achieved down to spacings of

$$
\Delta \mathrm{R}=1.22 \lambda / \theta_{\mathrm{i}}
$$


This holds even if the scattering reaching the detector is predominantly coherent [11], since the detector averages over the diffraction pattern. In most cases however, thermal diffuse scattering is the dominant means of scattering to the annular detector. Thermal vibrations destroy the long-range positional correlations within each column, so that each atom sees only a few neighbours within its column as correlated [12]. The length of such a "packet" depends on detector angle and Debye-Waller factor, but is generally less than the thickness of a typical specimen. In this case the intensity will be dependent simply on the number of packets in the column, ie. proportional to thickness $t$ (ignoring for the moment effects due to channelling and absorption which are discussed in the next section). Columns that are much shorter than this correlation length will scatter predominantly coherently, so that the intensity will be proportional to $t^{2}[11]$.

With $\mathrm{X}$-ray microanalysis, clearly we again collect a small but representative fraction of the total emission, but for electron energy loss spectroscopy (EELS), the strongly forward-peaked scattering makes it experimentally feasible to collect most of the scattering with modern aberration corrected spectrometers. Incoherent imaging conditions are again fulfilled [10], and the inelastic image is given by a convolution of the probe intensity profile with an inelastic object function centered on the atomic sites. However, because of the low characteristic scattering angles associated with low energy edges, the corresponding object functions may be substantially broader than the probe profile, so that to achieve atomic resolution a certain minimum energy loss is necessary as discussed in section 4 . 
It is clear that the conditions for incoherent imaging with elastically and inelastically scattered electrons are very complementary, so that both may be achieved simultaneously with the STEM geometry. The intensity in the electron optically limited probe is however significantly reduced from the conditions generally used for microanalysis, so that use of an efficient parallel detection system for EELS is essential. The Oak Ridge system uses a McMullan detector [13], based on a Wright Instruments multi-phase-pinned CCD camera, which provides exceptional sensitivity. Indeed, calculations indicate that single atom detection is feasible with this system [14], despite the low beam currents, due to the fact that we are illuminating only a few tens of atoms in one atomic column. A single atom therefore represents a much higher concentration than in situations where beam broadening is important, such as glasses or biological specimens [15]. Due to the likelihood of beam damage affecting the spectral fine structure, an important advantage of the McMullan system is its ability to record a sequence of spectra in rapid succession. Comparing the first spectrum with the last enables an assessment to be made of the importance of beam damage, and if there is none, the spectra may be summed to improve statistics. At present, only the $100 \mathrm{kV}$ STEM is equipped with this EELS capability.

Although no $\mathrm{X}$-ray microanalysis has yet been demonstrated at atomic resolution, the only limitation would appear to be the need for longer counting times with the increased possibility of beam damage. Although the EELS fine structure is the most sensitive indicator of beam damage, amorphization of a column is quite apparent from the Z-contrast image alone, and it would be reasonable to assume that if the image were not changed at the end of the analysis, then at least the major constituents of the 
column were not displaced during the analysis. X-ray analysis requires the use of the virtual objective aperture, and due to the high demagnification of the illumination system, smaller apertures than usual are required. The optimum angle can be adjusted by varying the condenser and objective lens strengths; extra demagnification can be achieved if necessary by increasing the strength of the gun lens. In this way, $Z$-contrast images can be obtained of equal quality to those obtained with the real objective aperture.

\section{Probe Channelling}

No consideration has so far been given to the effects of dynamical diffraction on the conditions needed to establish incoherent imaging. The reason for this is that dynamical diffraction manifests itself in a particularly simple way when a coherent probe is incident on a zone axis crystal. It has the effect of focussing the current onto the atomic columns, while maintaining the overall envelope of the incident probe, as indicated schematically in Fig. 2. For an incident probe size below the column spacing, most of the current can be channelled along a single atomic column, which is critical for Z-contrast imaging and microanalysisatomic at atomic resolution. Incoherent imaging is no longer restricted to weak phase objects, but applies also to crystals of substantial thickness.

This behaviour was apparent in early calculations of STEM probe propagation $[16,17]$, and its explanation lies in the effects of quantum mechanical superposition [18-20]. The STEM probe is a coherent superposition of plane waves emanating from the objective aperture, and is best thought of as a spherical wave converging on the specimen surface. Inside the crystal, the 
probe becomes a coherent superposition of fast electron Bloch states, and it is clear that if the amplitude and phase of a state is strongly dependent on incident angle, then superposition of states over a wide angular range will lead to destructive interference. The only states that survive this angular integration are those that are tightly bound to the atomic columns, since their amplitude and phase in reciprocal space is slowly varying. In $\mathrm{Si}$ and $\mathrm{Ge}$ for example, only $1 \mathrm{~s}$ states are important, and the probe therefore takes on the form of these states as it propagates into the crystal, becoming concentrated into narrow spikes around each atomic column.

With only 1s states contributing to the probe amplitude, then it is also clear that interference between different Bloch states becomes a second order effect, so that the usual oscillatory depth dependence of diffraction intensities will be suppressed to a large degree. For the same reason, beam broadening will also be strongly suppressed. The channelled flux propagates along the columns in the s states until it is scattered out to plane wave states by thermal vibrations, whereupon beam broadening is initiated in the usual way (Fig. 3). The penetration length depends on the s state absorption coefficient, which, since s states are in close proximity to the atomic nuclei, is quite strong and very sensitive on atomic number. At $100 \mathrm{kV}$ the flux can penetrate hundreds of Ångstroms in $\mathrm{Si}$, but only a few tens of Ångstroms along heavy columns. At $300 \mathrm{kV}$ penetration lengths are considerably enhanced. Such effects are well described in the Bloch wave description through an Einstein model of thermal vibrations, and the effect can be incorporated quantitatively in the appropriate cross section for imaging or microanalysis. If $\sigma_{i}(R)$ is the appropriate atomic cross section for the elastic or inelastic scattering process being detected, the columnar cross section is given by 


$$
O(R)=\sum_{i} \sigma_{i}(R) \varepsilon^{1 s^{2}} b^{1 s^{2}}(\mathbf{R})\left(\frac{1-e^{-2 \mu 1 s_{t}}}{2 \mu^{1 s_{t}}}\right)
$$

where the sum is over all atoms $i$ in the column, $b^{1 s}$ is the 1 sloch state of excitation $\varepsilon^{1 \mathrm{~s}}$ and absorption coefficient $\mu^{1 \mathrm{~s}}$, calculated for exact zone axis incidence. The angular dependence of these quantities is included by defining an effective probe intensity profile,

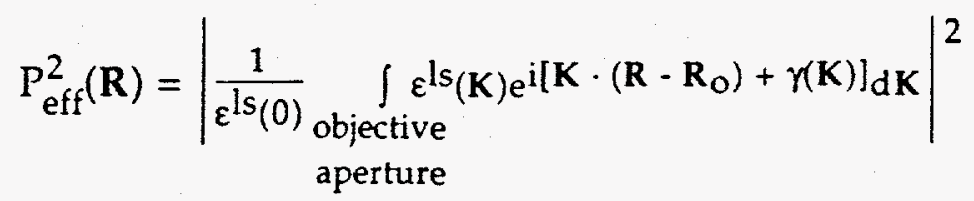

where $\mathbf{K}$ is the transverse component of the incident wave vector and

$$
\gamma=\frac{\pi}{\lambda}\left(\Delta \mathrm{f} \theta^{2}+\frac{1}{2} C_{s} \theta^{4}\right)
$$

is the transfer function phase factor for a defocus $\Delta f$ and electron wavelength $\lambda$. If the Bloch wave excitation $\varepsilon^{1 s}$ was constant over the aperture, this would be identical to the incident probe profile. Typically, $\varepsilon^{1 \mathrm{~s}}$ falls to about half its axial value at the edge of the objective aperture, which has the effect of broadening the effective probe by about $10 \%$ [19]. It can be appreciated that the constancy of $\varepsilon^{1 s}$ over the objective aperture is critical to forming a probe inside the crystal. $\varepsilon^{2 s}$ for example changes sign across the aperture, which is the reason that the $2 s$ Bloch state, although highly excited on axis, does not contribute significantly to the probe intensity inside the crystal. The Zcontrast, EELS or X-ray image is then given by the convolution of the effective probe with the columnar cross section, which can be considered as the object function for incoherent imaging: 


$$
\mathrm{I}\left(\mathbf{R}_{\mathbf{o}}\right)=\int \mathrm{P}_{\text {eff }}^{2}\left(\mathbf{R}-\mathbf{R}_{\mathbf{o}}\right) \mathrm{O}(\mathbf{R}) \mathrm{d} \mathbf{R} .
$$

\section{Localization of Inelastic Scattering}

Object functions for elastic scattering to the high angle detector are highly localized about the atomic columns, but this is not necessarily the case for inelastic scattering. Plasmons and low loss excitations may be quite delocalized, and would not carry atomic resolution chemical information. (Note that atomic resolution images in plasmon losses can be obtained through elastic scattering mechanisms, a situation usually referred to as preservation of contrast. This is unrelated to the present discussion which is concerned with the localization of the microanalytical information.) A good description of the object function for inelastic processes is therefore desirable. The object function for a transition from an initial state 10$\rangle$ to a final state $|n\rangle$ may be written in terms of the matrix elements $\rho_{\text {no }}$ as

$$
\mathrm{O}(\mathbf{R})=\left(\frac{\mathrm{e}^{2}}{\pi \hbar v}\right)^{2}\left|\int \frac{\rho_{\mathrm{no}}}{\mathrm{q}^{2}}(\mathbf{q}) \mathrm{e}^{-\mathrm{i} \mathbf{K} \cdot \mathbf{R}} \mathrm{dK}\right|^{2},
$$

$[10,21]$ where the momentum transfer $q=\left(\dot{q}_{z \min }, K\right)$, with $q_{z m i n}$ corresponding to the minimum momentum transfer at zero scattering angle. The object function can also be written in terms of a form factor in reciprocal space

$$
\mathrm{O}(\mathbf{R})=\frac{1}{(2 \pi)^{2}} \int \mathrm{f}^{\prime}(\mathbf{K}) \mathrm{e}^{\mathrm{i} \mathbf{K} \cdot \mathbf{R}} \mathrm{d} \mathbf{K}
$$

where

$$
f^{\prime}(K)=\left(\frac{e^{2}}{2 \pi^{2} h v}\right) \int \frac{\rho_{n o}\left(q^{\prime}\right)}{q^{\prime 2}} \frac{\rho_{n o}^{*}\left(q^{\prime}+K\right)}{\left|q^{\prime}+K\right|^{2}} d K^{\prime}
$$


Although this form factor has a similar angular dependence to the more familiar generalized oscillator strength, it is not identical. For high resolution microanalysis we will primarily be concerned with inner shell excitations, for which a hydrogenic model has been developed by Maslen and Rossouw [2226]. In this model, an inelastic excitation with scattering vector $\mathbf{q}$ for the fast electron is accompanied by ejection of a secondary electron with wavevector $\kappa$. As the secondary electron is not detected, $\kappa$ must be integrated coherently over all secondary electron emission directions, and the matrix product $\rho_{\text {no }}\left(q^{\prime}\right) \rho_{\text {no }}\left(q^{\prime}+K\right)$ replaced with

$$
\int \rho_{\text {no }}\left(q^{\prime}, \kappa\right) \rho_{\text {no }}^{*}\left(q^{\prime}+K, \kappa\right) d \kappa
$$

Convenient analytical expressions have been given for these integrals $[27,28]$. Fig. 4 . shows object functions calculated using the hydrogenic model assuming collection of all the scattering for edge energies appropriate for the Si-K loss $(1840 \mathrm{eV})$, Si-L loss $(100 \mathrm{eV})$ and the Co-L loss $(786 \mathrm{eV})$. Although the hydrogenic model was developed for $\mathrm{K}$ shells, we believe it to be the only model for inner shell excitations available at this time, and is likely to provide a more accurate description than simple estimates based on characteristic scattering angles [29-31]. The full width half maxima are $0.24 \AA$ for the Si K loss, $4.9 \AA$ for the Si L loss and $2.0 \AA$ for the Co L loss. Clearly one could not expect atomic resolution from the Si L loss, although it should be possible with the other two edges. Note the sensitive dependence of the object function width on atomic number, the Co $\mathrm{L}$ object function being almost an order of magnitude wider than that of the Si K edge, with less than a factor of two difference in energy. 
The corresponding functions in reciprocal space, $f^{\prime}(\mathbf{K})$, are shown in Fig. 5. Clearly, for our detection angle of $30 \mathrm{mrad}$, almost all of the scattering is indeed detected for the Co-L and Si-L edges, so that the conditions for incoherent imaging are fulfilled. This is not the case for the Si K loss, but provided the detector angle is much larger than the probe forming aperture, incoherent imaging still applies, and the real space object function is now given by restricting the integration in Eqn. 11. to the collector aperture

$$
\mathrm{O}(\mathbf{R})=\frac{1}{(2 \pi)^{2}} \int \mathbf{f}^{\prime}(\mathbf{K}) \mathrm{e}^{\mathrm{i} \mathbf{K} \cdot \mathbf{R}} \mathrm{d} \mathbf{K}
$$

The effect of a finite collector aperture on the object function for the Si K loss is shown in Fig. 6. Clearly, provided we ensure that our collection angle is significantly greater than the objective aperture (semiangle $\sim 10 \mathrm{mrad}$ ), then the use of a finite collector aperture will not cause a loss in resolution.

Note that all object functions show significant tails in real space, unlike the Gaussian forms often assumed. This will result in a non-local component to the image. It would be expected that this component could be removed by the use of an annular collector aperture to restrict low angle scattering from entering the spectrometer. Fig. 7. shows the significant resolution improvement to be expected with the Si L loss, a FWHM of $3.6 \AA$ and $2.8 \AA$ being achieved for an annular collection apertures of radius 2-30 mrad and 430 mrad respectively, which may be sufficient to allow atomic resolution to be achieved in Si. Larger inner collection angles cause substantial loss in intensity. 


\section{Atomic Resolution Spectroscopy at a $\mathrm{CoSi}_{2} / \mathrm{Si}(111)$ Interface}

The $\mathrm{CoSi}_{2} / \mathrm{Si}(111)$ epitaxial interface is an ideal test specimen for demonstrating atomic resolution since it can be grown atomically flat and abrupt as seen in the Z-contrast image in Fig 8 . This specimen was grown by high dose ion implantation and annealing, and shows an unexpected interface structure in that the last plane of the $\mathrm{Si}$ is seen to be in a twinned orientation [32]. The image was taken with the $100 \mathrm{kV} \mathrm{STEM}$, and from the profiles of Fig. 2a would not be expected to resolve the $1.36 \AA$ separation of the dumbbells, but instead each is imaged as a single elongated feature. In the $\mathrm{CoSi}_{2}$, the image is dominated by the high scattering power of the Co columns, and the Si columns are unresolved, forming a high background intensity to the image. Note that, due to the twin, the separation of the last plane of the $\mathrm{Si}$ and the first plane of the $\mathrm{CoSi}_{2}$ is only $2.7 \AA$.

Given the object function shown in Fig. 4. we would anticipate achieving atomic resolution using the Co L-loss. The spectra shown in Fig. 9. were obtained by centering a line scan over each plane in turn using the Z-contrast signal displayed on an oscilloscope, then acquiring a Co-L edge for 5 seconds [33]. In this way, the possibility of beam damage is minimised and any specimen drift is visible by observing the line scan. The use of a large collection aperture does degrade the energy resolution of the fine structure, the width of the $\mathrm{L}_{3}$ edge being about $5 \mathrm{eV}$. Thus precision in spatial information must be traded for precision in spectral detail due to averaging over a large range of momentum transfers. It is quite apparent that the Co edge drops dramatically in moving a single atomic plane across the interface. 
The specimen thickness in this region was estimated at $100-200 \AA$, so that no significant beam broadening should occur in the $\mathrm{Si}$, but some is to be expected for the heavier Co columns. A quantitative profile was obtained by least squares fitting to reference spectra taken several units cells from the interface, and resulted in the profile shown in Fig. 10. The inelastic intensity drops from $86 \%$ to $7 \%$ in a distance of $2.7 \AA$, demonstrating that atomic resolution is indeed achieved. Also shown is the expected profile for a $2.2 \AA$ probe and a perfectly localized inelastic scattering potential. The observed profile is clearly somewhat broadened as would be expected from the significant width of the object function. The asymmetry in the experimental profile is consistent with the anticipated onset of beam broadening in the silicide.

\section{Conclusions}

Microanalysis at atomic or near-atomic resolution is now experimentally feasible [33-36], opening up new areas of materials science to fundamental study, such as the mechanisms of segregation and embrittlement. The simultaneous acquisition of imaging and spectroscopic data is particularly powerful, due to the complementary nature of their information. For example, in a recent study of tilt grain boundaries in $\mathrm{SrTiO}_{3}, \mathrm{Z}$-contrast imaging and EELS were combined to deduce structure models directly from the experimental data [37-39]. Heavy columns were located by a maximum entropy analysis of the $Z$-contrast image to an accuracy of $\sim 0.2 \AA$, while the oxygen coordination was determined from the EELS fine structure. The importance of this capability was the finding of an unexpected structure, involving two half columns (or equivalently a single zig-zag column) at the core of each grain boundary dislocation. Such cores appeared in all structural 
units found, and appear to be a very effective way to avoid self-ion repulsion in ionic materials. Also, such columns represent attractive sites for the segregation of dopant atoms, which can now be investigated experimentally by these techniques. Furthermore, the model structures found in this way represent the perfect starting point for theoretical studies, by $a b$ initio methods for example. Both theory and experiment may now study the same systems in parallel.

In the future we can also expect to see $X$-ray analysis carried out at atomic resolution, adding complementary information on elements that are difficult to detect by EELS. We can also expect these techniques to be used with the 300 $\mathrm{kV}$ STEM, its smaller probe allowing smaller spaced columns to be illuminated individually, in intermetallics for example. Although the width of the EELS object function increases at the higher accelerating voltage, it does so slower than the probe size decreases, so that real advantages are to be anticipated for materials analysis.

\section{Acknowledgments}

We are grateful to J. T. Luck, T. C. Estes and S. L. Carney for technical assistance. This research was sponsored by the Division of Materials Sciences, U.S. Department of Energy, under contract No. DE-AC05-84OR21400 with Martin Marietta Energy Systems, Inc. 


\section{Figure Captions}

1. Probe intensity profiles as a function of defocus for (a) the $100 \mathrm{kV} \mathrm{HB} 501$ UX and (b) the $300 \mathrm{kV} \mathrm{HB} 603$ at Oak Ridge National Laboratory.

2. Schematic indicating the columnar channelling of a coherent probe in a zone axis crystal.

3. Schematic indicating the reduction in beam broadening as a result of columnar channelling.

4. Object functions for the $\mathrm{Si} \mathrm{K}, \mathrm{Si}-\mathrm{L}$ and $\mathrm{Co}-\mathrm{L}$ losses, calculated on the hydrogenic model for a $50 \mathrm{eV}$ ejected secondary electron and assuming all scattered electrons are collected. Plots have been normalised to their peak intensity to emphasize differences in width. Note the object function for $\mathrm{Si}-\mathrm{L}$ is negative at $\mathrm{r}=0$.

5. Corresponding object functions in reciprocal space.

6. Reduction and broadening of the $\mathrm{Si}-\mathrm{K}$ object function due to the use of a finite collection aperture.

7. Enhanced spatial resolution expected with the Si-L loss using an annular collection aperture. Note the significant enhancement of the negative central part of the object function, which will cause loss in signal intensity.

8. Z-contrast image of an epitaxial $\mathrm{CoSi}_{2} / \mathrm{Si}(111)$ interface showing the last layer of $\mathrm{Si}$ to be in a twinned orientation.

9. Co L spectra recorded plane by plane across the interface shown in Fig. 8 .

10. Profile showing integrated Co edge intensity plane by plane across the interface (crosses). The sharp drop in Co intensity on moving a single plane across the interface demonstrates atomic resolution. Circles show the theoretical profile for a localized object function. 


\section{References}

1. L. M. Brown, Nature 366 (1993) 721,

2. A. V. Crewe, J. Wall and J. Langmore, Science 168, (1970) 1338

3. A. V. Crewe and J. Wall, J. Molec. Biol.. 48 (1970) 375

4. A. V. Crewe, J. P. Langmore, and M. S. Isaacson, in: Physical Aspects of Electron Microscopy and Microbeam Analysis, eds. B. M. Siegel and D. R. Beaman (Wiley and Sons, New York, 1975), p. 47.

5. O. Scherzer, J. Appl. Phys. 20 (1949) 20.

6. A. V. Crewe, Rep. Prog. Phys.. 43 (1980) 621.

7. A. V. Crewe and D. B. Salzman, Ultramicroscopy. 9 (1982) 373.

8. C. Colliex and C. Mory, in Quantitative Electron Microscopy, eds. J. N. Chapman and A. J. Craven (Scottish Universities Summer School in Physics, Edinburgh, Scotland, 1984) p. 149.

9. S. J. Pennycook, D. E. Jesson and N. D. Browning, Nucl Instr and Meth in press (1995)

10. R. H. Ritchie and A. Howie, Phil Mag A 58, (1988) 753

11. D. E. Jesson and S. J. Pennycook, Proc RSoc Lond A441, (1993) 261

12. D. E. Jesson and S. J. Pennycook, Proc RSoc Lond in press (1995)

13. D. McMullan, J. M. Rodenburg, Y. Murooka, and A. J. McGibbon, Institute of Physics, Conference.Series, 98, (1990) 55.

14. N. D. Browning, and S. J. Pennycook, Microbeam Analysis 2, (1993) 81.

15. R. D. Leapman, in Transmission Electron Energy Loss Spectrometry in Materials Science, eds. M. M. Disko, C. C. Ahn and B. Fultz, The Minerals, Metals \& Materials Society, (1992) p. 47.

16. J. Fertig and H. Rose, Ultramicroscopy 2 (1977) 269.

17. R. F. Loane, E. J. Kirkland, and J. Silcox, Acta Cryst. A44 (1988) 912. 


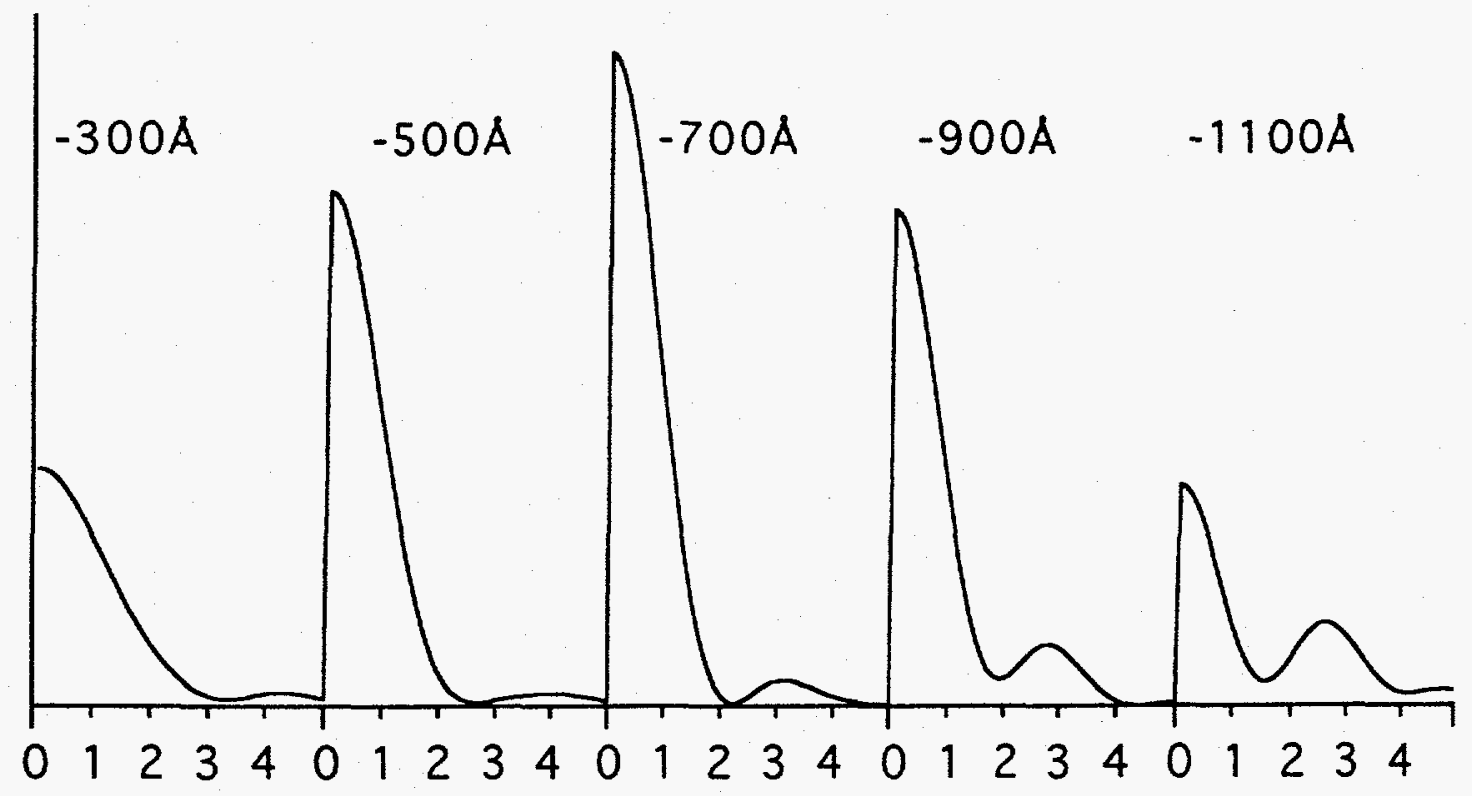




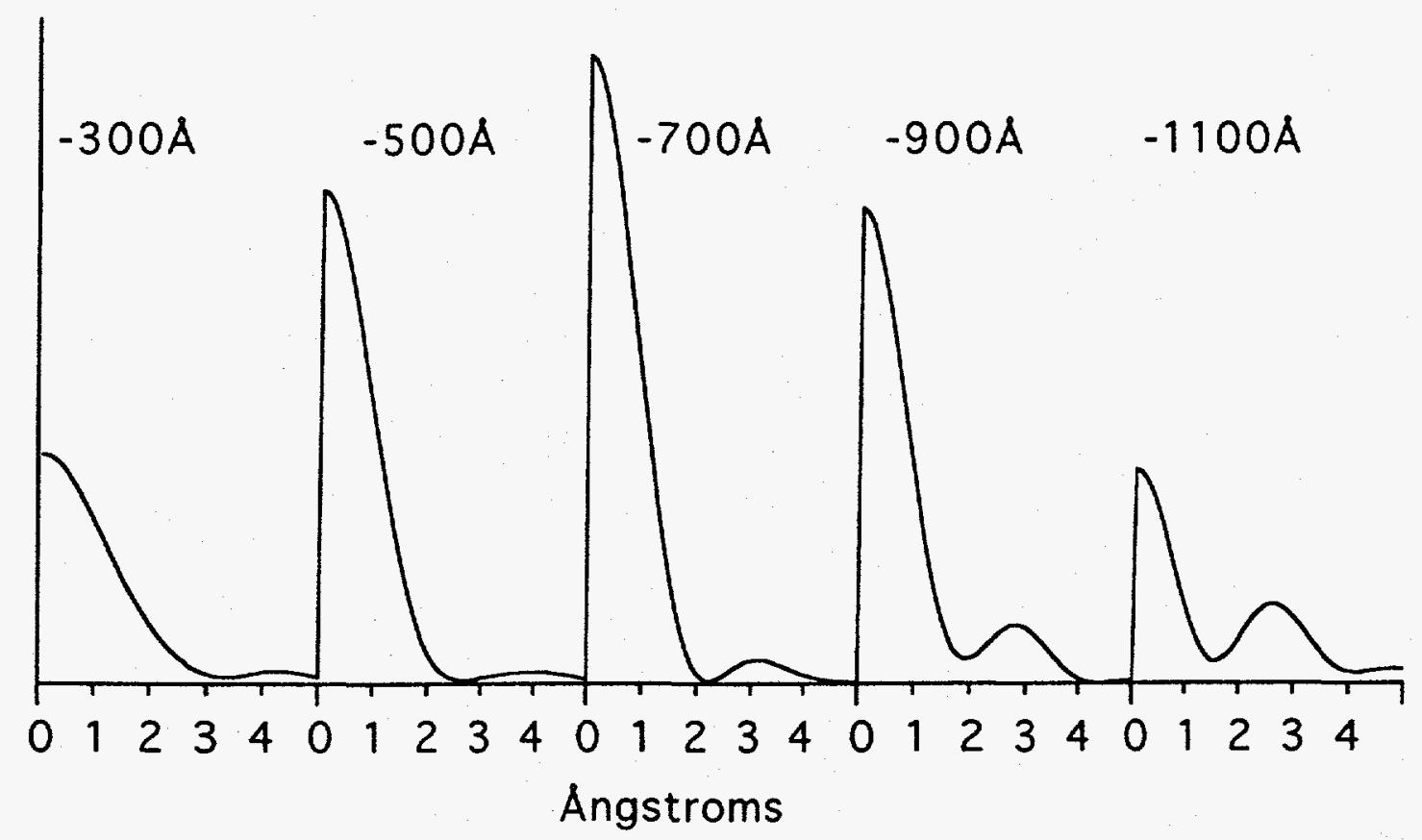




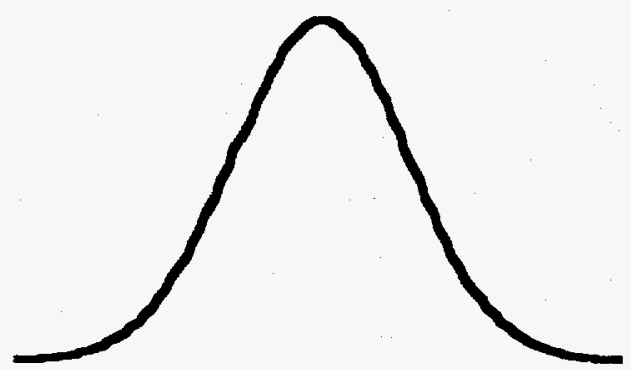

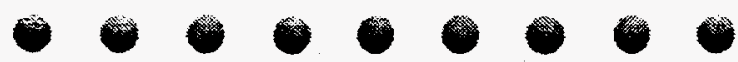

0000000

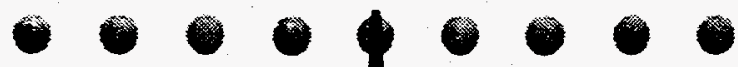

- $0 \% 000$

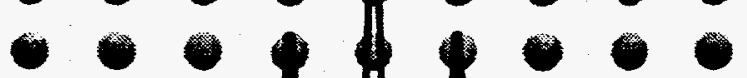

- 0 f 1000

- 8189.

- dagado。

- 0 - 0.0

a

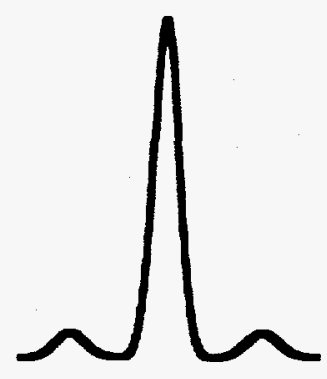

- 0.0 - 0000 - 1000 $-0000$ - 01000 - 0 - 0 - 1.0 - - a 10 - 0000

b 


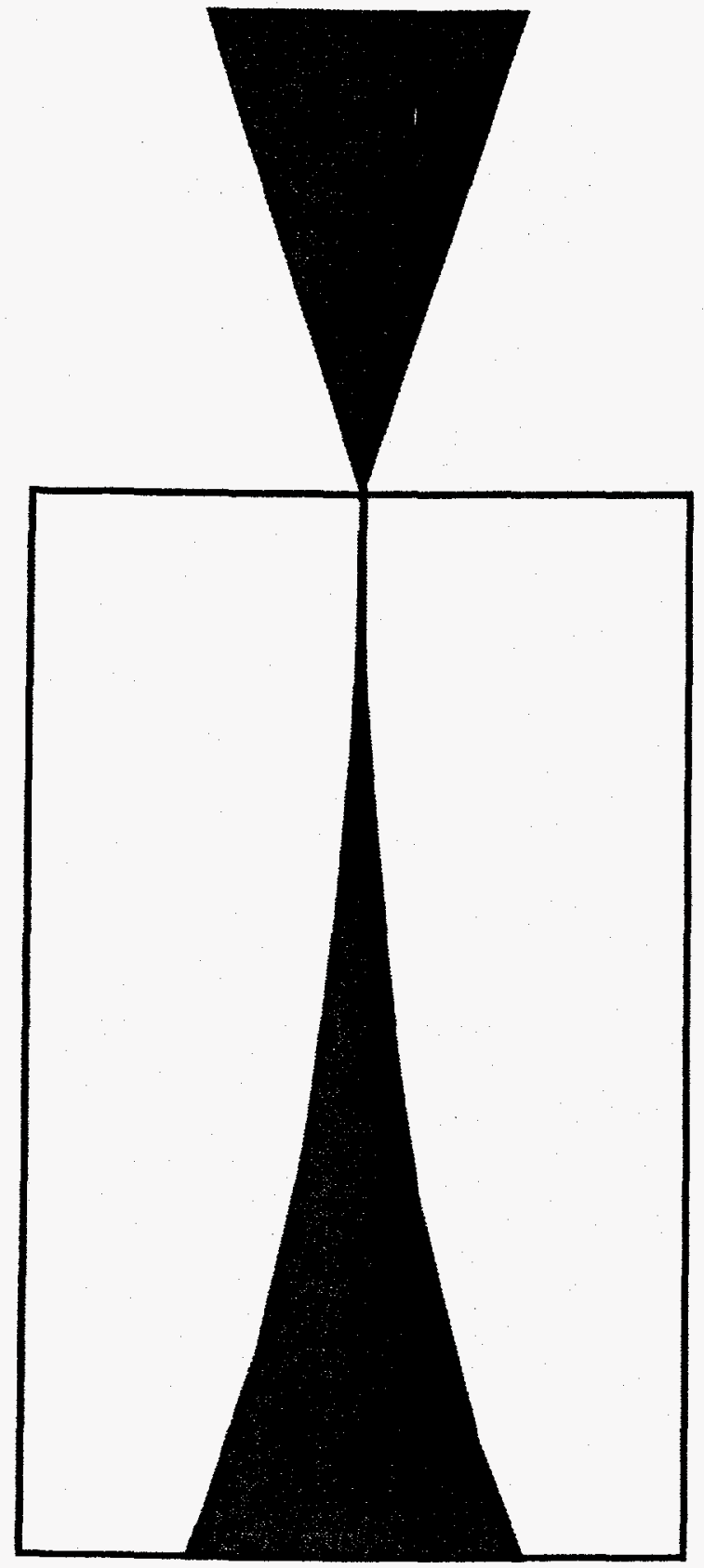

a

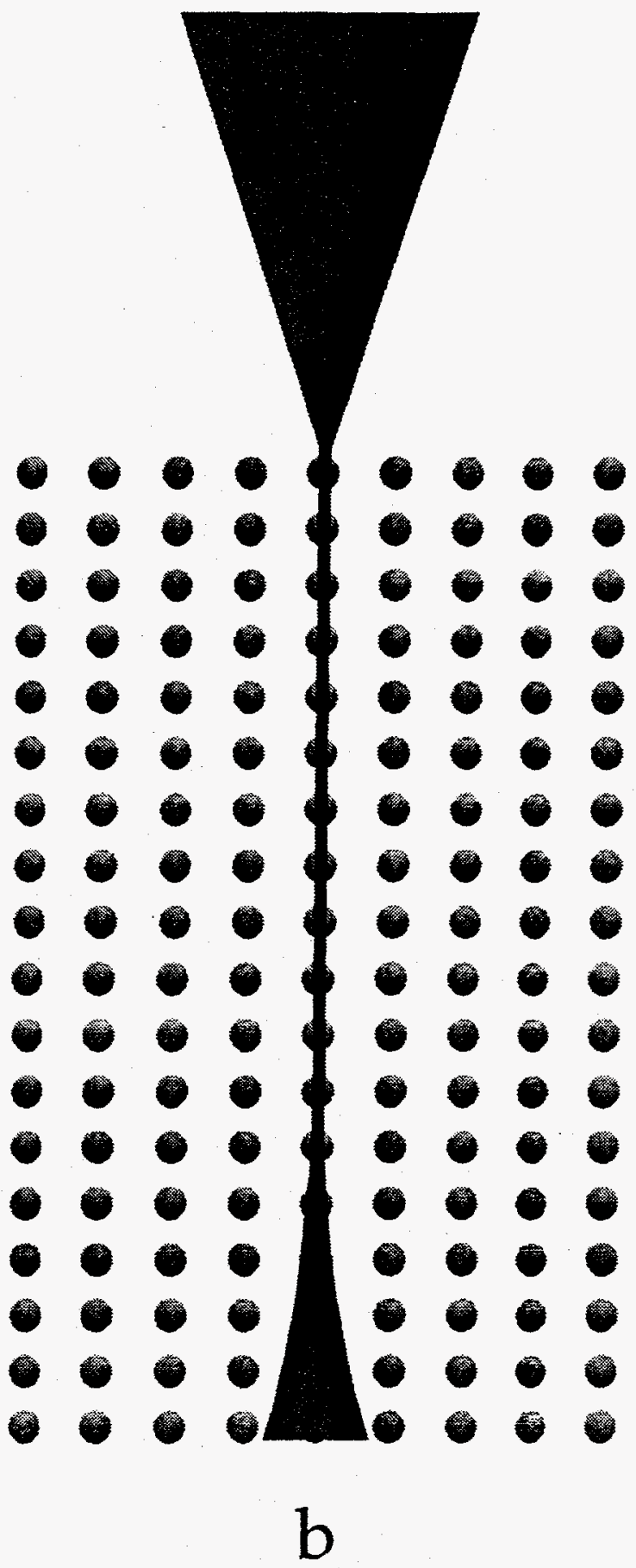

b 


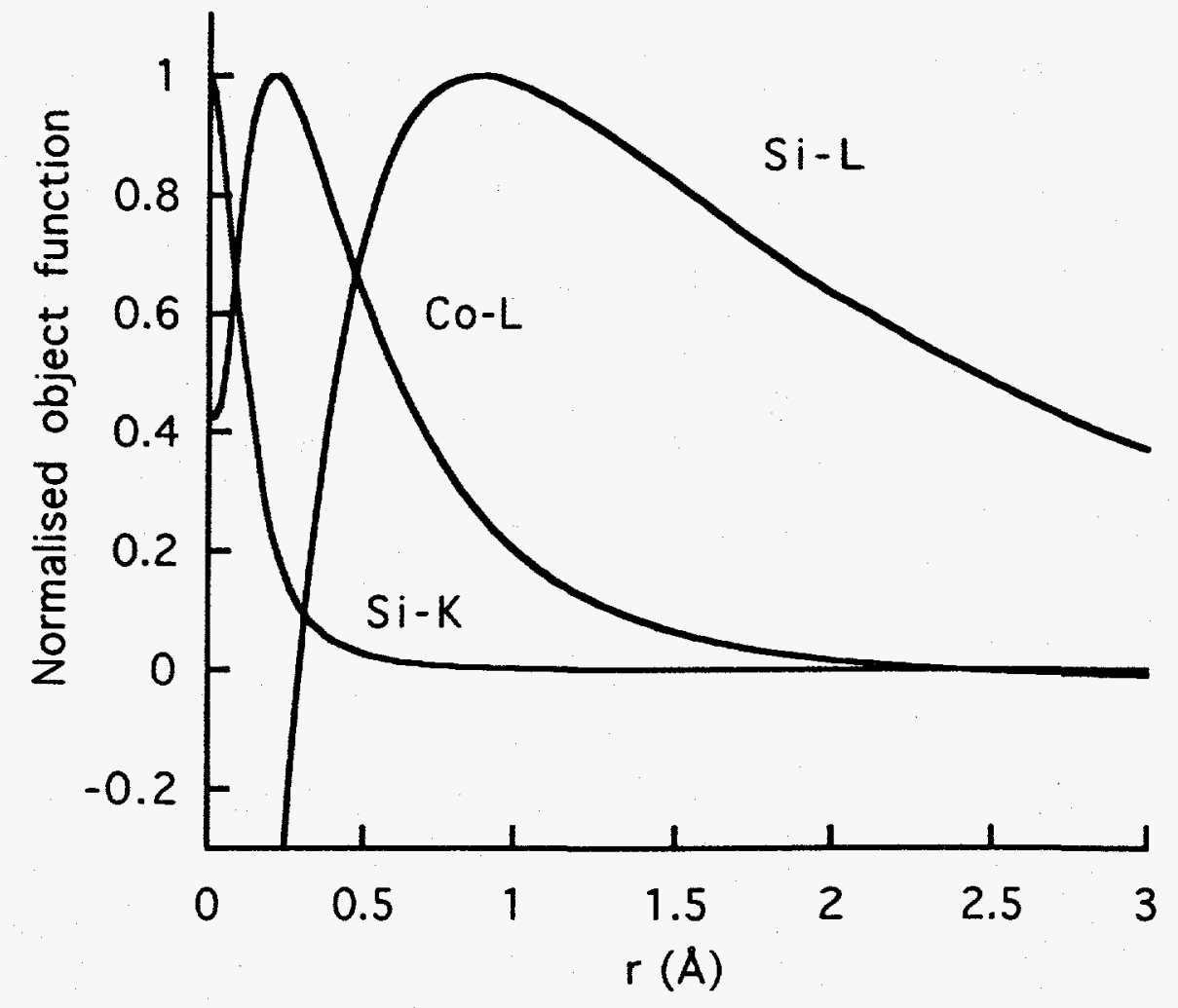




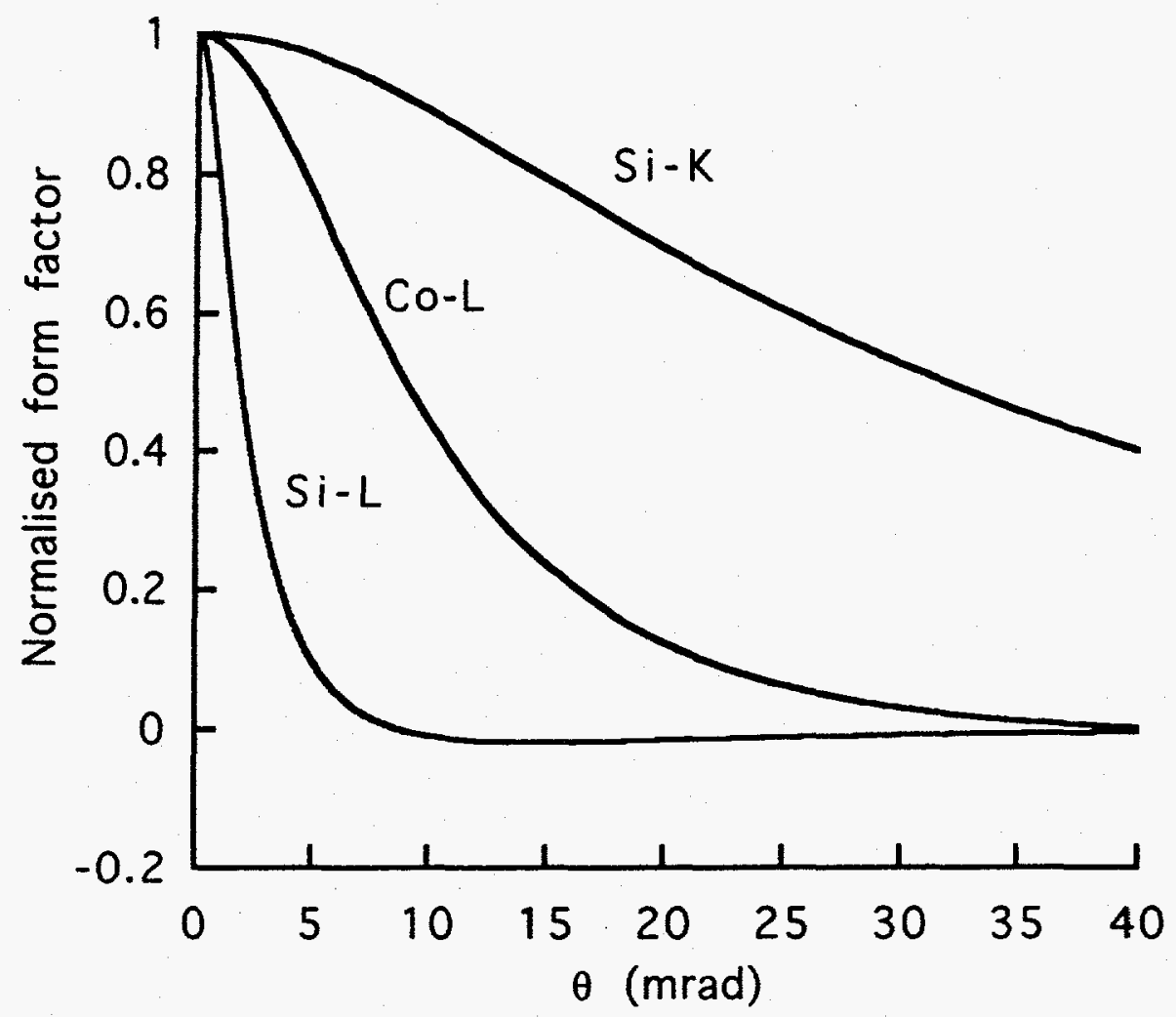




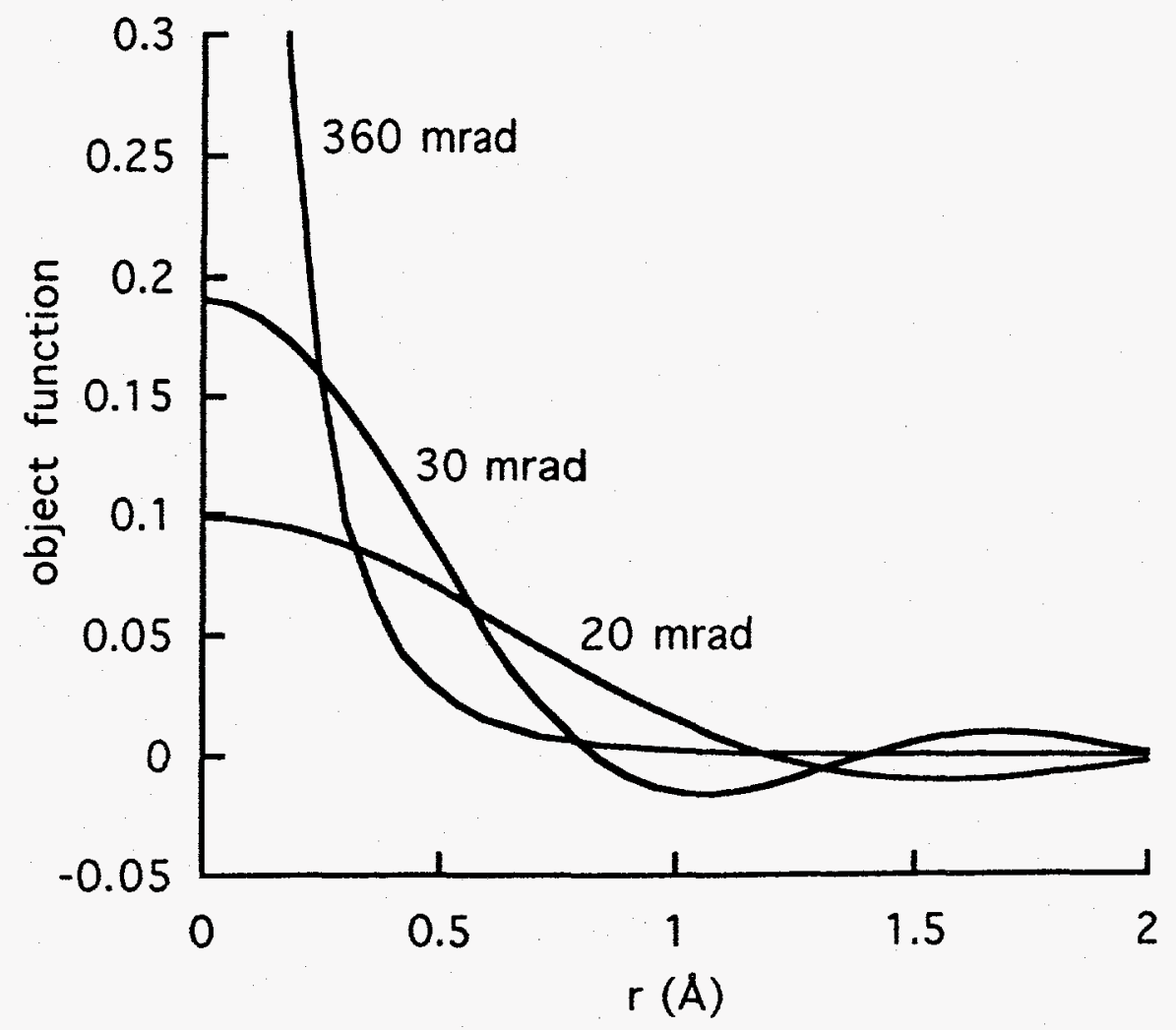




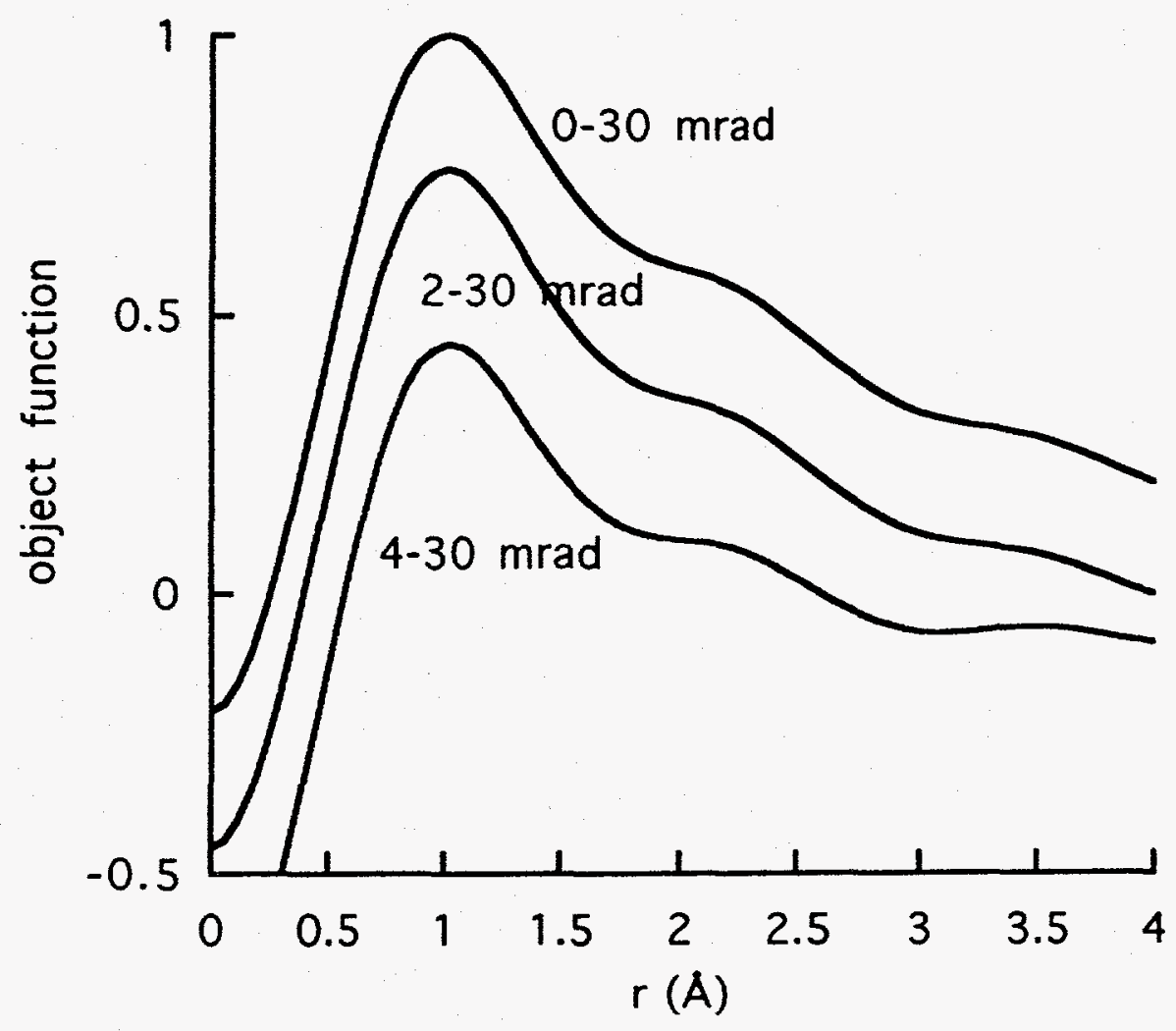




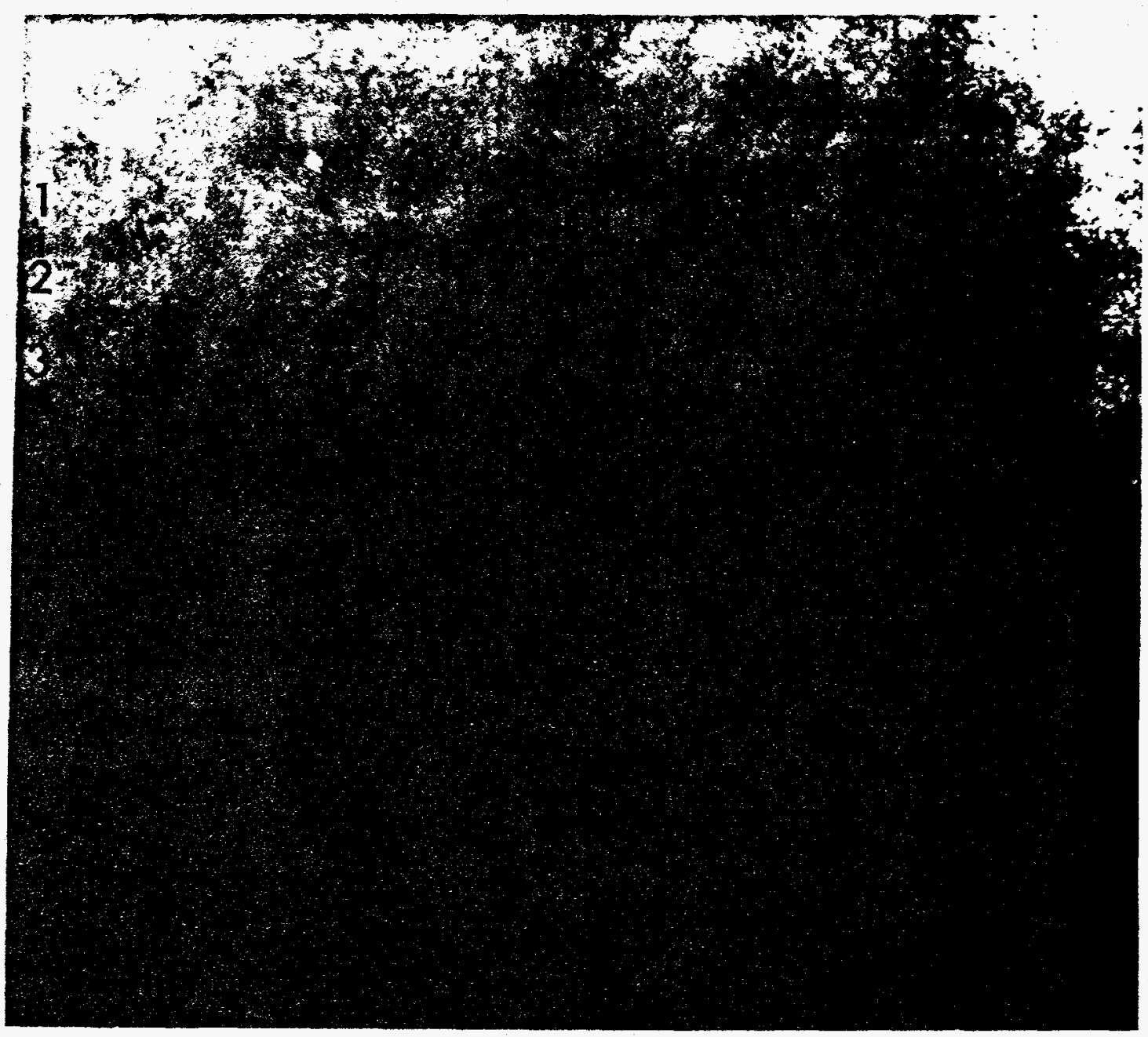




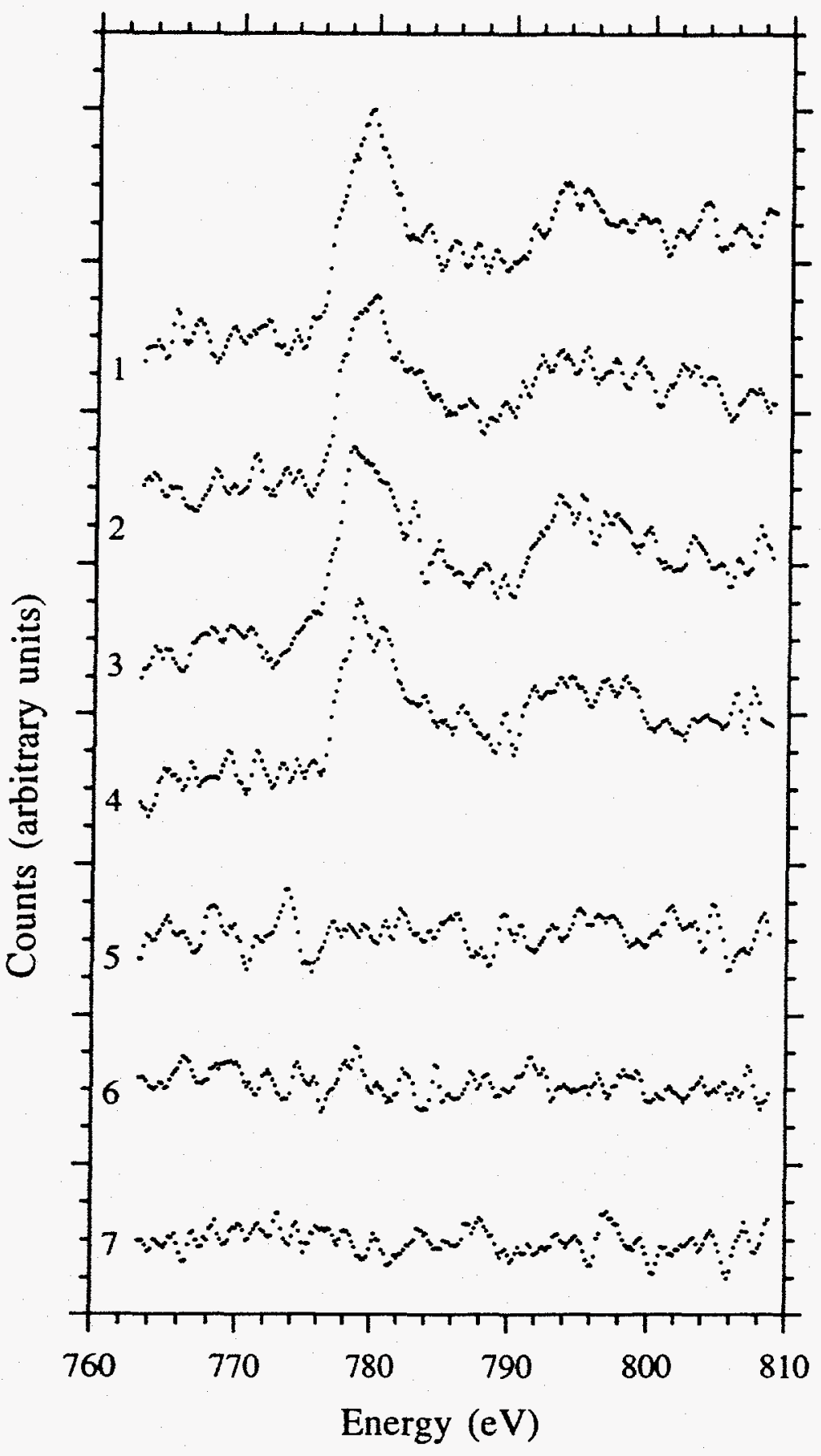




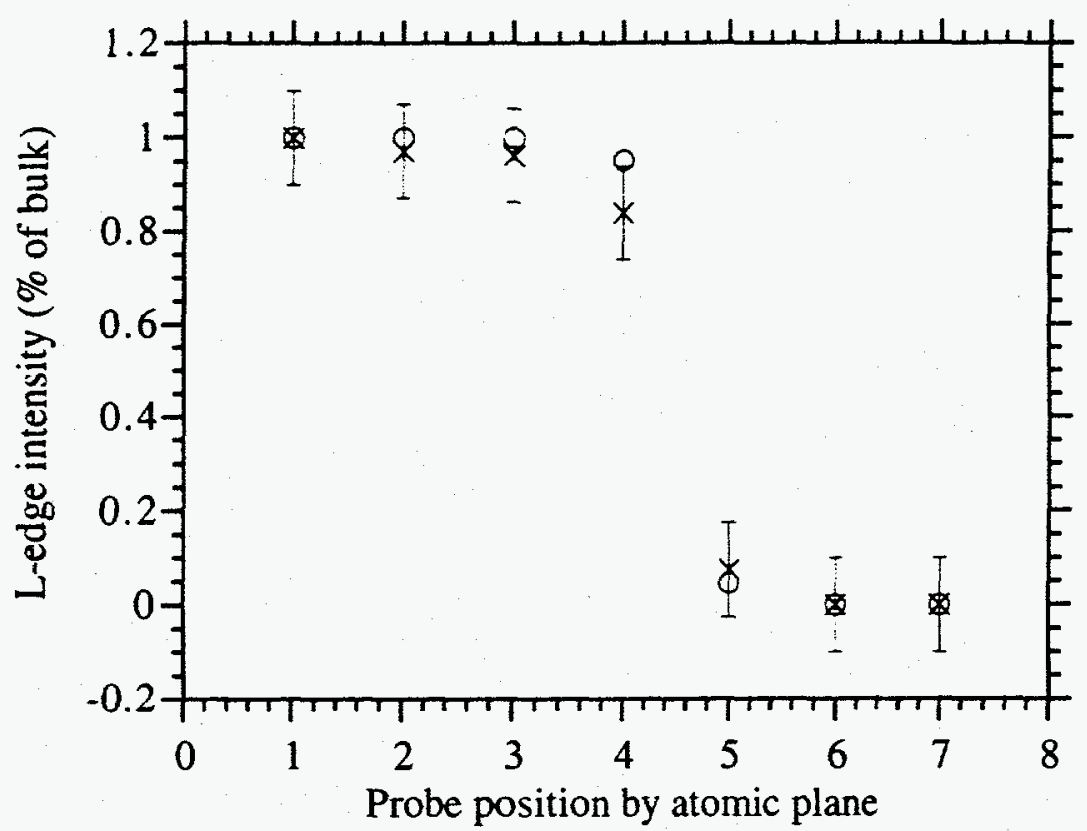

